\title{
Occurrence of Vibrational resonance in an oscillator with an asymmetric Toda potential
}

\author{
Olusola Kolebaje $^{\mathrm{a}, \mathrm{c}}$, O. O. Popoola ${ }^{\mathrm{b}}, \mathrm{U}$. E. Vincent ${ }^{\mathrm{c}, \mathrm{d}, *}$ \\ a Department of Physics, Adeyemi College of Education, 350106, Ondo, Nigeria \\ ${ }^{\mathrm{b}}$ Department of Physics, University of Ibadan, Ibadan, Nigeria. \\ ${ }^{\mathrm{c}}$ Department of Physical Sciences, Redeemer's University, P.M.B. 230, Ede, Nigeria. \\ d Department of Physics, Lancaster University, Lancaster LA1 4YB, United Kingdom.
}

\begin{abstract}
Vibrational resonance (VR) is a phenomenon wherein the response of a nonlinear oscillator driven by biharmonic forces with two different frequencies, $\omega$ and $\Omega$, such that $\Omega \gg \omega$, is enhanced by optimizing the parameters of high-frequency driving force. In this paper, an counterintuitive scenario in which a biharmonically driven nonlinear oscillator does not vibrate under the well known VR conditions is reported. This behaviour was observed in a system with an integrable and asymmetric Toda potential driven by biharmonic forces in the usual VR configuration. It is shown that with constant dissipation and in the presence of biharmonic forces, VR does not take place, whereas with nonlinear displacement-dependent periodic dissipation multiple VR can be induced at certain values of high-frequency force parameters. Theoretical analysis are validated using numerical computation and Simulink implementation in MATLAB. Finally, the regime in parameter space of the dissipation for optimum occurrence of multiple VR in the Toda oscillator was estimated. This result would be relevant for experimental applications of dual-frequency driven laser models where the Toda potential is extensively employed.
\end{abstract}

Key words: Oscillations, Vibrations, resonance, Nonlinear dissipation, Toda oscillator

\section{Introduction}

Since Toda [12/3/4 proposed an exponential interaction lattice between particles, now known as the Toda potential more than forty decades ago to explain the phenomenon 5 of self-pulsation, a quasi-periodic pulsation of the output intensity of a solid-state laser in the transient regime, the ${ }^{25}$ Toda potential has gained enormous research attention due to its several applications in optics engineering. The major important properties of the Toda potential are its inte10 grability, the existence of periodic and stable solitary wave solutions; as well as exact solutions for the dynamics and ${ }^{30}$ the statistical thermodynamics [5/6/7. The Toda potential is also of special interest because it is the simplest potential function with an Henon type integral or constant of motion and interaction acts only between the neighbouring particles 8 . The aforementioned properties makes the ${ }^{35}$ Toda lattice system one of the most prominent subject of research focus, even in the recent times. Indeed, earlier

\footnotetext{
* corresponding author.

Email address: u.vincent@lancaster.ac.uk (U. E. Vincent).
}

studies employed the Toda potential or lattice for modent heat baths at the ends [10, and the molecular dynamics of muscle contraction 11. Some new soliton solutions to the Toda lattice were obtained using a modified bilinear Bäcklund transformation [12, Darboux transformation 1314, exponential-function method 15, hyperbolic function method 16, and extended tanh-function approach, with the asymptotic stability proved in 17 . The Toda lattice has been shown to be super-integrable because for every $N$ degrees of freedom, it possesses $2 N-1$ independent constants of motion 7 . In addition, numerical solution for the Toda lattice has been obtained via the Variational Iteration Method [18, and Adomian Decomposition Method [19].

More pertinent to the present study, Toda lattice has been successfully used to model different classes of lasers such as a damped an-harmonic oscillator subject to the Toda potential (Otto and Politi 20), modulated lasers 2122, class-B lasers 2324] and bad-cavity lasers 25126]. Cialdi et al. 24] confirmed experimentally that the Toda os40 cillator model describes excellently the early stage dynam- 
ics of the Nd:YAG laser. Investigations on the dynamics of the Toda oscillator has revealed several intriguing dynamical behaviours including sub- or supercritical Andronov-100 Hopf bifurcation born from stable or unstable branch of 45 periodic solutions, period doubling, saddle-node, NeimarkSaker bifurcations, fold-bifurcations, chaos and hyperchaos in coupled antiphase driven Toda oscillators [27 28 29 30]. In all the previous studies, the Toda oscillators were drivem05 mostly by a single sinusoidal force. In the paper by Kim et was reported in a dual-frequency driven Toda oscillator at two incommensurate frequencies. However, to the best of our knowledge there is no report on the occurrence of vibra-110 tional resonance (VR) 32 in dual-frequency driven Toda oscillator

Motivated by the foregoing, we investigate the phenomenon of vibrational resonance (VR), first studied by Landa and McClintock 32] - manifesting in nonlinear sys-115 tems driven by a biharmonic external force with two different frequencies $\omega$ and $\Omega$; a low-frequency force $F \cos \omega t$ and a high-frequency force $G \cos \Omega t[33$. The amplification of the weak input signal by the biharmonic periodic external force carries significant importance in communications 34 35, laser physics 36, acoustics 37, medicine 38, neuroscience [39], geosciences [40, and ecology [41]. Following the work of Landa and McClintock 32, Gitterman 42. and Blekhman and Landa 43] developed theoretical techniques for analysing VR. Consequently, the occurrence of VR has been investigated theoretically and numerically in monostable systems 444546, multistable systems 44, excitable systems [47, and time-delayed systems [48, asymmetric Duffing oscillator 4649, biological nonlinear ${ }_{125}$ maps [50, the quintic oscillator [445], a bistable system 51, gene transcriptional regulatory system 5253; and a bichromatic excitation [54, nonlinearly-damped oscillators [55 56 57], asymmetrical deformable oscillator [58], in time-delay gene transcriptional regulatory system [59] and in systems with rough potentials 60 , to mention but $\mathrm{a}^{130}$ few. The majority of the above-mentioned results demonstrated the effects and roles of the high-frequency force parameters $G$ and $\Omega$ on the occurrence of VR. Recently, the effect of depth and location of the potential well on VR was reported in a quintic oscillator 61. Furthermore, contributory roles in the occurrence of VR 55 56 57. Very recently, a new body of research in connection with the VR phenomenon in the quantum domain has emerged 6263]. In addition, a recent review presented a new formalism for

90 describing VR in position-dependent mass systems [64.140 The wide incidence of VR observed in many kinds of system are linked to its potential applications in, for instance, detection of weak random signals [65], improving weak aperiodic signals [66], amplification of an auto-dyne signals in signal with arbitrary high-frequencies 6869 , detection ofi45 weak signals in the presence of strong background noise
[70.

In this paper, we report the result of an analysis on possible occurrence of VR in an oscillator with an integrable and asymmetric Toda potential. We will show, counterintuitively, that under the actions of dual-frequency signal forces, the Toda oscillator with constant dissipation coefficient does not vibrate. However, with periodic dissipation in place, VR is induced. We employed both analytical and numerical treatments to investigate the mechanism of the VR and provide clear evidence that introducing periodicity into the otherwise constant damping coefficient can induce VR in the presence of the dual-frequency driving force. The present result differs significantly from previous reports in which nonlinear dissipation enhances vibrationinduced resonances as reported in [55 56 57]. The paper is organized as follows: the model is introduced in Section 2 . The theoretical and numerical analysis of VR are presented in Section 3 and 4 respectively with the discussions and conclusion provided in Section 5 .

\section{The model}

The model is a generalized damped and bi-harmonically driven nonlinear system with a Toda potential. The equation of motion is given by 31

$\frac{d^{2} x}{d t^{2}}+\nu(x) \frac{d x}{d t}+\frac{d U}{d x}=F \cos \omega t+G \cos \Omega t$,

where $F \cos \omega t$ is the low-frequency force with frequency $\omega$ while $G \cos \Omega t$ is the high-frequency force with frequency $\Omega$; and $\Omega>>\omega$. In Eq. (11), we have included a $2 \pi$-periodic multiplicative displacement-dependent damping term, $\nu(x)$ which is a function of $k_{0}$ and $\epsilon$ denoting, respectively the damping amplitude and the strength of the dissipation in the form

$\nu(x)=k_{0}(1+\epsilon \cos x) ;$

while $U(x)$ is the well known Toda potential given by

$U(x)=e^{x}-x+1$.

The Toda potential has an extremely asymmetric curvature and a linear dependence on the displacement $x$ in the $x<0$ region when $\lim _{x \rightarrow-\infty} U(x)=-x+1$, which is equivalent to free fall dynamic. In the region $x>0$, i.e. when $\lim _{x \rightarrow \infty} U(x)=e^{x}$ the system contains a very hard spring. In the analysis that will follow, we will show that the displacement-dependent periodic damping is essential for the occurrence of VR and that in its absence, the Toda oscillator will not vibrate even in the presence of dualfrequency forcing. Using Eq. (2) and Eq. (3) in Eq. (1), the system to be analysed becomes

$\ddot{x}+k_{0}(1+\epsilon \cos x) \dot{x}+e^{x}-1=F \cos \omega t+G \cos \Omega t$.

When $\epsilon=0$, system (2) reduces to the exact model of a quasiperiodically forced Toda oscillator exhibiting nontrivial intermittent route to strange nonchaotic attractors 
(SNAs) reported in 31. Furthermore, if only one external driving force is considered for constant damping, i.e.175 $\epsilon=0$, system (2) reduces to the well investigated driven

Toda oscillator exhibiting abundant varieties of bifurcation structures including period-doubling, devil's staircase and chaos [27. This system possesses unique asymmetry that is at variance with oscillators in Duffing family. In fact, its arguably, a good prototypical and simplest model for asymmetric oscillators; and as such has been used to model ${ }^{180}$ a nonlinear RLC circuit 71. Figures 1 and 2 illustrate damping in Eq. (4). The amplitude and location of local minima and maxima of the periodic damping depends on the sign of the damping amplitude $k_{0}$ and strength of inthe salient features of the Toda potential and the periodic homogeneity $\epsilon$.

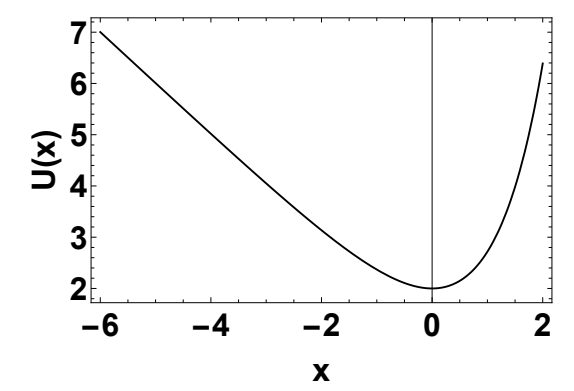

Fig. 1. Shape of the Toda Potential $U(x)=e^{x}-x+1$.
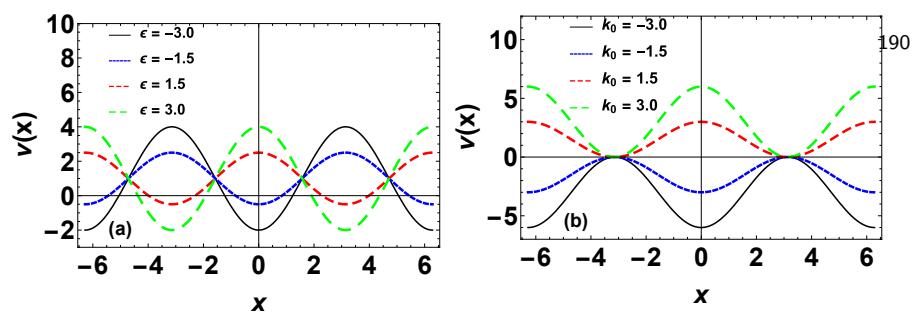

Fig. 2. Shape of the Periodic Damping Term $\nu(x)$ for; (a) four values of $\epsilon$ with $k_{0}=1$ (b) four values of $k_{0}$ with $\epsilon=1$.

\section{Theoretical Analysis}

To examine VR theoretically, we employ the method of separation of motion to separate Eq. (4) into slow and fast motion. Since $\Omega \gg \omega$, we can assume that the solution ofoo Eq. (44) is of the form $x(t)=X(t)+\psi(t, \tau=\Omega t)$, where $X$ and $\psi$ are the slow motion with frequency $\omega$ and period $2 \pi / \omega$ and fast motion in fast time $\tau$ with frequency $\Omega$ and period $2 \pi / \Omega$, respectively. The mean value of $\psi$ with respect to time $\tau$ is given by

$\langle\psi\rangle=\frac{1}{2 \pi} \int_{0}^{2 \pi} \psi(\tau) d \tau=0$.

Substituting $x=X+\psi$ and Eq. (5) into Eq. (41), we obtain

$$
\begin{gathered}
\ddot{X}+\ddot{\psi}+k_{0}[1+\epsilon \cos X \cos \psi-\epsilon \sin X \sin \psi](\dot{X}+\dot{\psi}) \\
+e^{X+\psi}-1=F \cos \omega t+G \cos \Omega t .
\end{gathered}
$$

Since $\psi$ is a periodic function in fast time $\tau$, then by averaging both sides of Eq. (6) over the period $\left[0, \frac{2 \pi}{\Omega}\right]$, then the equation for the slow motion can be obtained as

$\ddot{X}+k_{0}[1+\epsilon \cos X\langle\cos \psi\rangle-\epsilon \sin X\langle\sin \psi\rangle] \dot{X}+e^{X}\left\langle e^{\psi}\right\rangle-1=F \cos$

The equation for the fast motion $\psi$ is obtained by subtracting Eq. (7) from Eq. (6) and by inertial approximation $\ddot{\psi}>>\dot{\psi}>>\psi$, it reduces to $\ddot{\psi}=G \cos \Omega t$, which has the solution

$\psi(\tau)=-\frac{G}{\Omega^{2}} \cos \tau$

With $\psi$ in Eq. (8), we obtain the mean values

$$
\begin{aligned}
& \langle\sin \psi\rangle=\frac{1}{2 \pi} \int_{0}^{2 \pi} \sin \psi(\tau) d \tau=0 \\
& \langle\cos \psi\rangle=\frac{1}{2 \pi} \int_{0}^{2 \pi} \cos \psi(\tau) d \tau=J_{0}\left(G / \Omega^{2}\right), \\
& \left\langle e^{\psi}\right\rangle=\frac{1}{2 \pi} \int_{0}^{2 \pi} e^{\psi(\tau)} d \tau=I_{0}\left(G / \Omega^{2}\right)
\end{aligned}
$$

where $J_{0}\left(G / \Omega^{2}\right)$ and $I_{0}\left(G / \Omega^{2}\right)$ are respectively the zeroth-order Bessel function of the first kind and the zeroth-order modified Bessel function of the first kind. Eq. (9) allows us to simplify Eq. (7), so that the equation for the slow motion becomes

$\ddot{X}+k_{0}\left[1+\epsilon J_{0}\left(G / \Omega^{2}\right) \cos X\right] \dot{X}+I_{0}\left(G / \Omega^{2}\right) e^{X}-1=F \cos \omega t .(1$

Eq. (10) can be re-written as the equation of motion of a system in the form

$\ddot{X}+\lambda_{e f f} \dot{X}+\frac{d V_{e f f}(X)}{d X}=F \cos \omega t$

where

$V_{e f f}=I_{0}\left(G / \Omega^{2}\right) e^{X}-X$

and

$\lambda_{e f f}=k_{0}\left[1+\epsilon J_{0}\left(G / \Omega^{2}\right) \cos X\right]$

are the effective potential and effective damping coefficient respectively. The shape and number of local minima and maxima of the effective potential depends on the parameters $G, \Omega$ and $\beta$. Figure 3 shows that the location of local minima changes with increasing $G$ and $\Omega$ respectively.

The effective potential of the system is always a single well with a minimum located at

$X^{*}=-\ln \left(I_{0}\left(\frac{G}{\Omega^{2}}\right)\right)$

The equation of motion for the deviation variable $Y=$ $X-X^{*}$ is given by

$\ddot{Y}+k_{0}\left[1+\epsilon J_{0} \cos Y+X^{*}\right] \dot{Y}+I_{0} e^{X^{*}} e^{Y}-1=F \cos \omega t .(15)$

$\ddot{Y}+k_{0}\left[1+\epsilon J_{0} \cos X^{*} \cos Y-\epsilon J_{0} \sin X^{*} \sin Y\right] \dot{Y}+e^{Y}-1=F \cos$ 

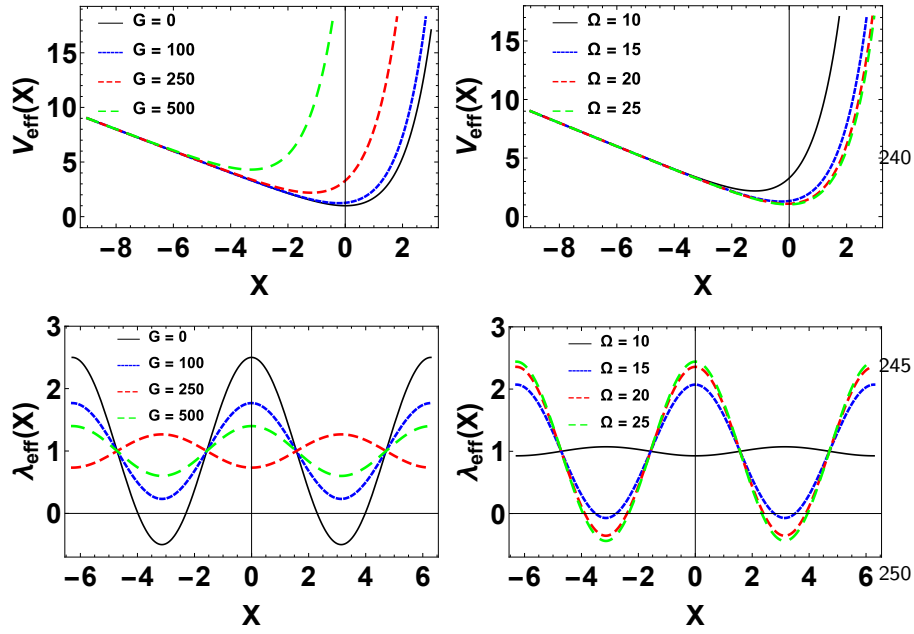

Fig. 3. The effective potential $V_{\text {eff }}(X)$ for (a) four values of $G$ with $\Omega=10$ (b) four values of $\Omega$ with $G=250$ and the effective damping $\lambda_{\text {eff }}(X)$ with $k_{0}=1, \epsilon=1.5$ for (c) four values of $G$ with $\Omega=10$ (d) four values of $\Omega$ with $G=250$.

For $F<<1$, then $|Y|<<1$ and we can approximate $\cos Y \approx 1, \sin Y \approx Y$ and $e^{Y} \approx 1+Y$, so Eq. (16) becomes $^{255}$

$\ddot{Y}+k_{0}\left[1+\epsilon J_{0} \cos X^{*}-\epsilon J_{0} \sin X^{*} Y\right] \dot{Y}+Y=F \cos \omega t$.

By neglecting the nonlinear terms in Eq. 17. we obtain an approximate damped and periodically forced linear equa- ${ }_{260}$ tion of the form

$\ddot{Y}+\lambda \dot{Y}+Y=F \cos \omega t$,

where

$\lambda=k_{0}\left[1+\epsilon J_{0}\left(\frac{G}{\Omega^{2}}\right) \cos \alpha\right]$

and $\alpha=\ln \left[I_{0}\left(\frac{G}{\Omega^{2}}\right)^{-1}\right]$. The solution of Eq. (18) in the long-time limit $(t \rightarrow \infty)$ is $Y(t)=A_{L} \cos (\omega t+\phi)$; where $A_{L}=F / \sqrt{S}$ and

$S=\left(1-\omega^{2}\right)^{2}+\lambda^{2} \omega^{2}, \quad \phi=\tan ^{-1}\left[\frac{\lambda \omega}{\omega^{2}-1}\right]$.

Hence, the response amplitude, which is the measure of amplification of the input signal by the high frequency signal

$Q=\frac{A_{L}}{F}=\frac{1}{\sqrt{\left(1-\omega^{2}\right)^{2}+\lambda^{2} \omega^{2}}}$.

Notably, for constant dissipation or damping, i.e. $\epsilon=0$, $\lambda$ in Eq. (19) reduces to $\lambda=k_{0}$. Consequently, theoreti 270 cal analysis based on the method of separation of motion suggests that, the Toda oscillator (1) does not admit VR for constant dissipation or damping on account that the response amplitude $Q$ in Eq. (21) is independent on the parameters $G$ and $\Omega$ of the high-frequency force. Remarkably, a variation in the response amplitude with the high- is sufficiently significant to be regarded as an amplification or de-amplification of the input signal is an open issue for further investigation. However, when $\epsilon$ takes on nonzero values multiple VR can occur in different $\epsilon$-regimes - which is the main result of this paper as we shall further illustrate. It is emphasized here that the non-occurrence of VR for constant dissipation is remarkably counterintuitive and at variant with results obtained from the Duffing oscillator family as well as all the previous results obtained other systems.

Now, let us analyze the occurrence of vibrational resonance as $G$ is varied. From Eq. (20), we see that $S_{G}=$ $2 \lambda \omega^{2} \lambda_{G}$, where $\lambda_{G}=d \lambda / d G$. Hence, VR is attained when $S_{G}=0$, which could be achieved subject to the following conditions: (i) $\lambda=0$ or (ii) $\lambda_{G}=0$ with $\lambda_{G G}=$ $d^{2} \lambda /\left.d G^{2}\right|_{G_{V R}}>0$.

Since $k_{0} \neq 0$, then $\lambda=0$ when

$\frac{1}{\epsilon}=-J_{0}\left(\frac{G}{\Omega^{2}}\right) \cos \alpha$

and the resonance value is given by $Q_{\lambda=0}=1 /\left|1-\omega^{2}\right|$, $\omega^{2} \neq 1$; which satisfies case (i). Hence, for fixed values of the other system parameters, the response amplitude $Q_{\lambda=0}$ is achieved at values of $G$ that satisfy Eq. (22). Figure 4 shows the values of $G$ for which $\lambda=0$ for selected values of $\Omega$ and $\epsilon$. Figure 4 shows that $\lambda=0$ only for $\epsilon$ values in the region $-1<\epsilon^{-1}<\epsilon_{0}^{-1}$ with multiple VR due to $\lambda=0$ occuring only for $\epsilon$ values in the region $\epsilon_{1}^{-1}<\epsilon^{-1}<\epsilon_{0}^{-1}$, where $\epsilon_{1}^{-1}=-0.3243$ and $\epsilon_{0}^{-1}=0.0935$. Also, the range of values of $G$ for which $\lambda=0$ increases as $\Omega$ decreases. That 65 is, resonance due to $\lambda=0$ occurs only for $\epsilon\langle-1$ and $\epsilon>$ $\epsilon_{0}$. Hence, for $-1<\epsilon<\epsilon_{0}$, VR will not occur since $\lambda=0$.
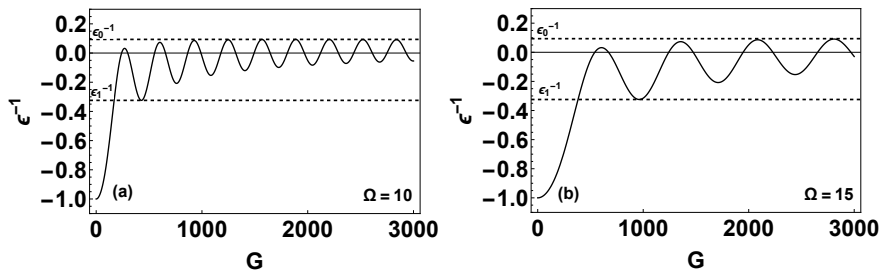

Fig. 4. The Plot of $1 / \epsilon$ against $G$ from Eq. (22) with (a) $\Omega=10$ and (b) $\Omega=15$

The second condition, i.e. $\lambda_{G}=0$ is such that

$\lambda_{G}=\frac{k_{0} \epsilon}{\Omega^{2}}\left[-J_{1} \cos \alpha+\frac{I_{1} J_{0}}{I_{0}} \sin \alpha\right]$

where $J_{1}$ and $I_{1}$ are respectively the Bessel function of the first kind of order 1 and the modified Bessel function of the first kind of order one. From Eq. (23), since $k_{0} \neq 0$, for $\lambda_{G}=0$ and $\lambda_{G G}>0$, then there are peaks in the response amplitude for $G$ that satisfy

$J_{1} \cos \alpha=\frac{I_{1} J_{0}}{I_{0}} \sin \alpha$.

The roots of Eq. (24), $G_{V R}$, occur at the critical points of $\lambda$. For $k_{0}>0, G_{V R}$ occur at the values of $\mathrm{G}$ for which $\lambda$ 
is at a local minimum. In addition, for $k_{0}<0, G_{V R}$ valuess20 at which VR occurs coincide with the local maxima of $\lambda$. The minima and maxima values of $\lambda$ are located at $G_{V R}$ that satisfy (24). It was observed that the oscillatory nature of the effective damping term $\lambda$ gives rise to oscillatory variation of the response amplitude $Q$ with VR taking place at the minima or maxima of $\lambda$ for $k_{0}>0$ and $k_{0}<0$ respectively. Thus, one can infer that the choice of damping nature would determine the nature of the response curve.

We remark that not all values of $G_{V R}$ due to $\lambda_{G}=0$ corresponds to $\mathrm{VR}$, since the peak produced in the $Q$-curve does not necessarily imply a gain in the response amplitude at $G=0$. The $Q$ value at $G_{V R}$ due to $\lambda_{G}=0$ corresponds to VR when $Q_{G_{V R}}>Q_{G=0}$ with

$Q_{G=0}=\frac{1}{\sqrt{\left(1-\omega^{2}\right)^{2}+\lambda_{G=0}^{2} \omega^{2}}}, \quad \lambda_{G=0}=k_{0}(1+\epsilon)$.

The $G$ values for which $\lambda_{G=0}$ produces a gain in the response amplitude at $Q_{G=0}$ is subject to the condition that: $\left|\lambda_{G_{V R}}\right|<\left|\lambda_{G=0}\right|$ is satisfied.
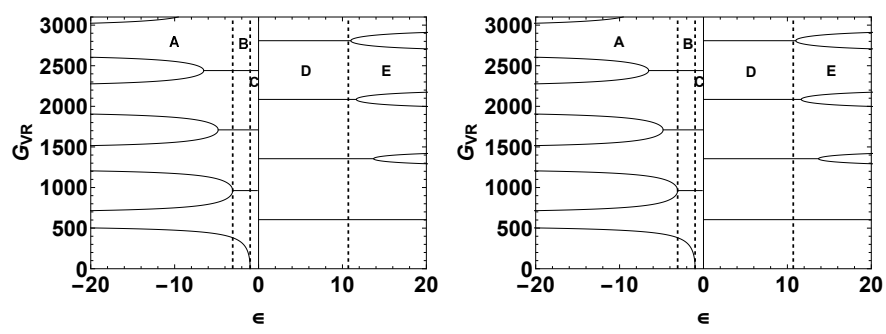

Fig. 5. Variation of the theoretically predicted $G_{V R}$ with the parameter $\epsilon$ for (a) $\Omega=10$ (b) $\Omega=15$. The vertical dashed lines are at $\epsilon=\epsilon_{1}=-3.08, \epsilon=-1$ and $\epsilon=\epsilon_{0}=10.70$.

In Figure [5] we plot the theoretically calculated $G_{V R}$ against the strength of inhomogeneity of the system $\epsilon$ for $\Omega=10$ and $\Omega=15$ respectively. The shape of the response curves can be predicted and understood from Figure 5 by analysing the Regions A-E of $\epsilon$ values.

(i) Region A $\left(\epsilon<\epsilon_{1}\right)$ : Multiple VR occurs because both conditions $\lambda_{G}=0$ and $\lambda=0$ are satisfied.

(ii) Region B $\left(\epsilon_{1}<\epsilon<-1\right)$ : VR occur only at one value of $G$ when $\lambda=0$ and at certain values of $G$ when $\lambda_{G}=0$, provided $\left|\lambda_{G_{V R}}\right|<\left|\lambda_{G=0}\right|$. number of $G_{V R}$ points decreases with increase in the highsignal frequency $\Omega$.
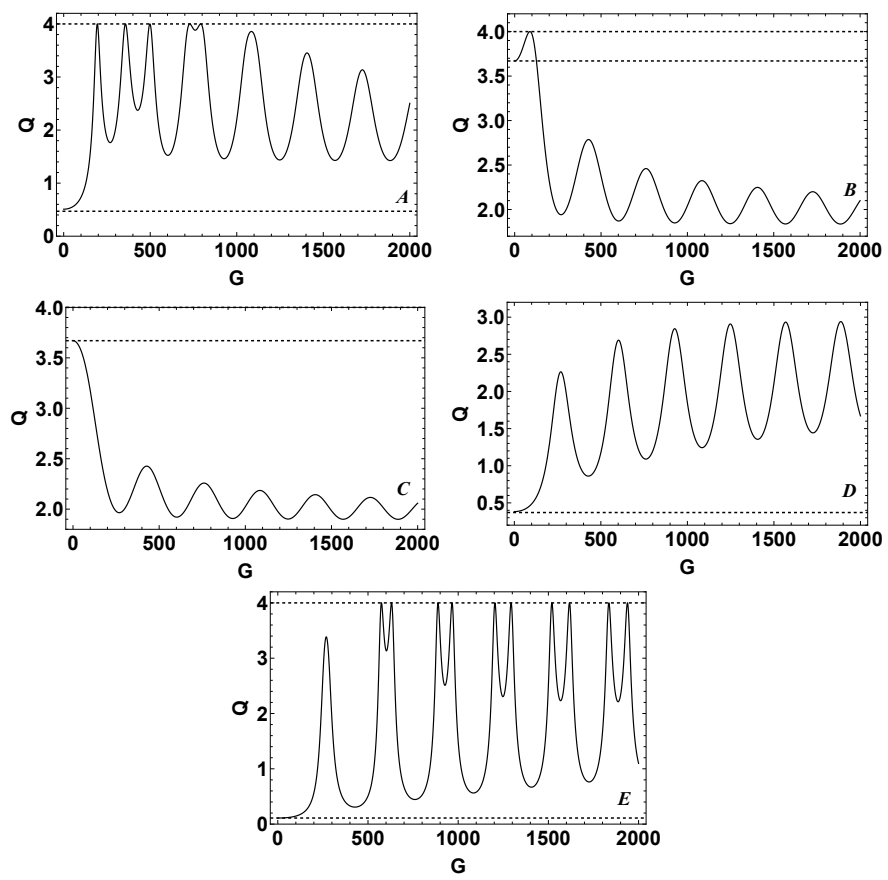

Fig. 6. Plot of theoretically computed response amplitude $Q$ as a function of $G$ for $\omega^{2}=0.75, k_{0}=0.5, \Omega=10$ and $\epsilon$ values in Regions A-E.
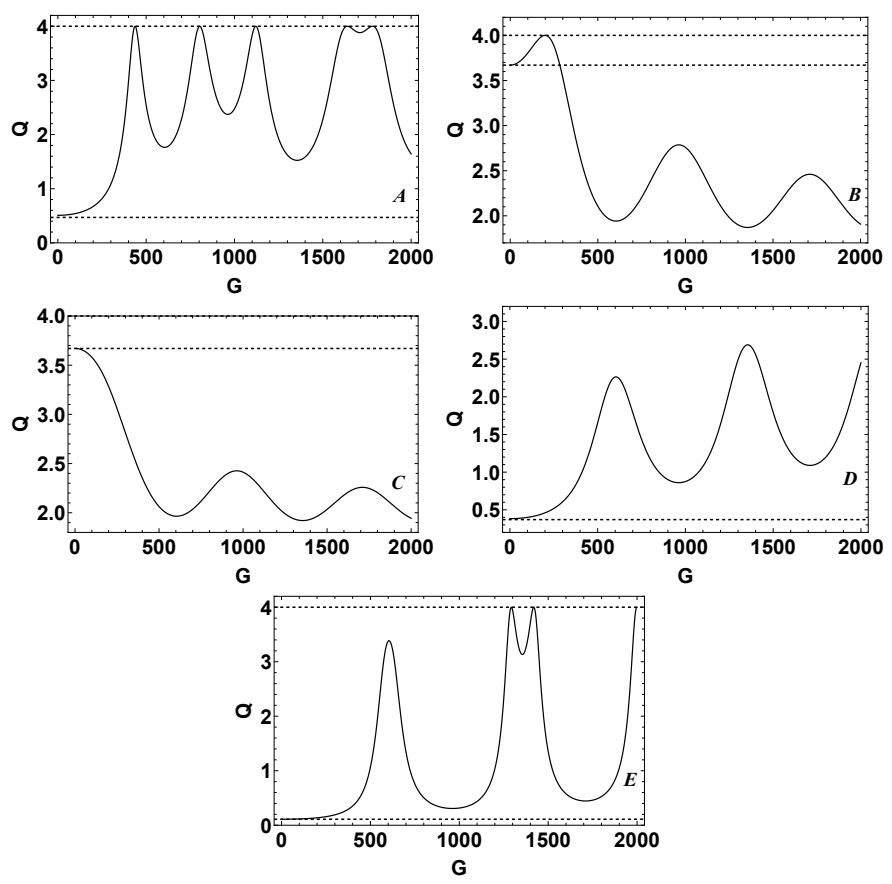

Fig. 7. Plot of theoretically computed response amplitude $Q$ as a function of $G$ for $\omega^{2}=0.75, k_{0}=0.5, \Omega=15$ and $\epsilon$ values in Regions A-E. 


\section{Numerical and Simulink Verification}

The second-order nonautonomous ordinary differential equation (ODE) given by Eq. (4) can be re-expressed as an70 equivalent system of two first-order autonomous ODEs of the form:

$$
\begin{aligned}
& \frac{d x}{d t}=y \\
& \frac{d y}{d t}=-k_{0}(1+\epsilon \cos x) y-e^{x}+1+F \cos \omega t+G \cos \Omega(26)
\end{aligned}
$$

Numerical integration of Eq. (26) is achieved via the fourth-order Runge-Kutta scheme with a suitable step size over the time interval $n T$ with $T=2 \pi / \omega$ being the period ${ }^{380}$ of the low-frequency force $F$ and $n$ the number of complete oscillations.

Following the numerical integration of Eq. (26), the response amplitude $Q$ can then be calculated numerically using the components of the Fourier spectrum of the time

$Q=\frac{A}{F}=\frac{\sqrt{Q_{S}^{2}+Q_{C}^{2}}}{F}$

where

$Q_{S}=\frac{2}{n T} \int_{0}^{n T} x(t) \sin \omega t d t, \quad Q_{C}=\frac{2}{n T} \int_{0}^{n T} x(t) \cos \omega t d t$

$n$ can be taken to be a suitably large value, say, 200 .

We first examine and compare theoretically and numerically obtained dependence of the response amplitude $Q$ on the damping amplitude $k_{0}$ and strength of inhomogeneity $\epsilon$. In Figure 8, we have presented plot of the response amplitude $Q$ as a function of the high-frequency force $G$, for various values of the strength of inhomogeneity $\epsilon$ at a fixed $k_{0}$ value; while in Figure 9, we present plot of the response amplitude $Q$ as a function of the high-frequency force $G$, for various values of the damping amplitude $k_{0}$ at a fixed $\epsilon$ value. The response amplitude $Q$ calculated from theory and numerics are represented by the continuous curve and marker points respectively. In Figure 8 , different $\epsilon$ values were chosen, i.e. $\epsilon=-2.5,1.5$ and 11.5 in different parameter regimes, namely, negative, low and high values of the damping inhomogeneity $\epsilon$. Figures 8 and 9 shows that there is good agreement between the theoretically and numerically obtained response $Q$. Moreover, the shape and trend of the theory (continuous curve) and numerics (solid marker points) are in good agreement. An increase in the value of 385 $\Omega$ leads to a decrease in the frequency of occurrence of the resonance points. With the variation in the damping amplitude $k_{0}$ at a fixed value of $\epsilon$, the high-frequency force $G$ values at which VR occurs remains unchanged. However, the position of the maxima and minima of the response ampli-390 tude $Q$ increases with decrease in the damping amplitude.

We implemented the Toda oscillator model given by Eq. (26) in MATLAB-Simulink. Simulink is a MATLAB- based environment for modelling and simulating dynamical systems. A block diagram of the system of equations was created in MATLAB-Simulink and is shown in Figure 10. Subsystem 1 in Figure 10 has input 1 and input 2 as $x$ and 1 respectively and an output that performs $k_{0}(1+\epsilon \cos x)$. Subsystem 2 is a time-input block with two outputs: output 1 and output 2 which represents the driving functions, $F \cos \omega t$ and $G \cos \Omega t$, respectively. The data obtained from the Simulink environment was plotted in MATLAB and are presented in Figures 11 and 12, Figures 11 and 12 show that the Simulink implementation of the Toda oscillator model given by Eq. (26) was in excellent agreement with the theoretically calculated response amplitude $Q$ given by Eq. (21).
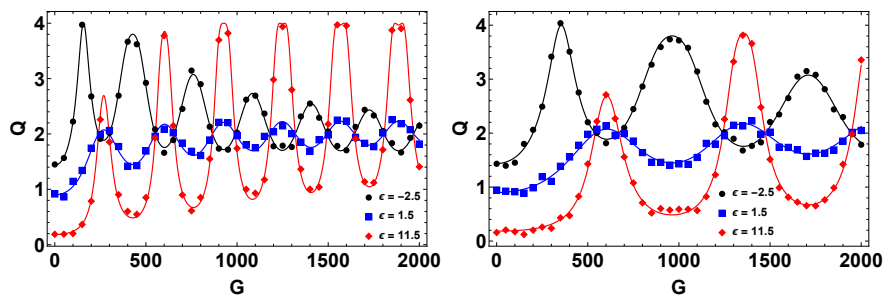

Fig. 8. Dependence of the response amplitude $Q$ on $G$ for three values of the damping amplitude $\epsilon=-2.5, \epsilon=1.5$ and $\epsilon=11.5$ with the other parameters fixed at $F=0.2, \omega^{2}=0.75, k_{0}=0.5, \Omega=10$ (left-panel) and $\Omega=15$ (right-panel). The continuous curve and solid markers represent the theoretically and numerically computed values of $Q$ respectively.
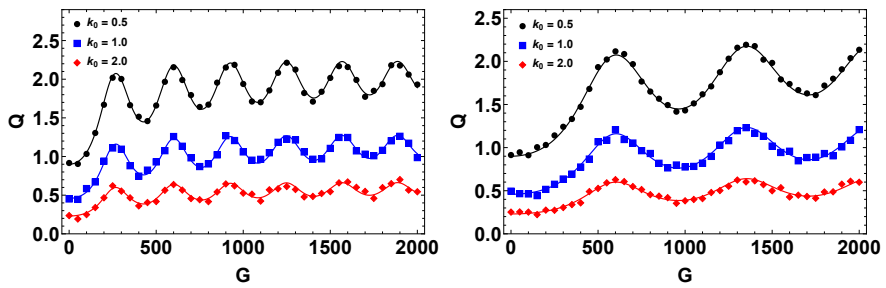

Fig. 9. Dependence of the response amplitude $Q$ on $G$ for three values of the damping amplitude $k_{0}=0.5, k_{0}=1.0$ and $k_{0}=2.0$ with the other parameters fixed at $F=0.2, \omega^{2}=0.75, \epsilon=1.5, \Omega=10$ (left-panel) and $\Omega=15$ (right-panel). The continuous curve and solid markers represent the theoretically and numerically computed values of $Q$ respectively.

\section{Conclusion}

In this paper, we have theoretically studied and numerically verified the occurrence of the phenomenon of vibrational resonance in a damped and bi-harmonically driven Toda oscillator model with asymmetric potential. Theoretical analysis of VR was carried out by first separating the model into fast and slow motions. The response amplitude was then obtained from the linearised equation of the slow motion. One of the most striking and remarkable observations was the non-occurrence of VR when the damping coefficient take on constant values. However with displacementdependent periodic damping, multiple resonances are induced for a wide range of the damping parameters. The 


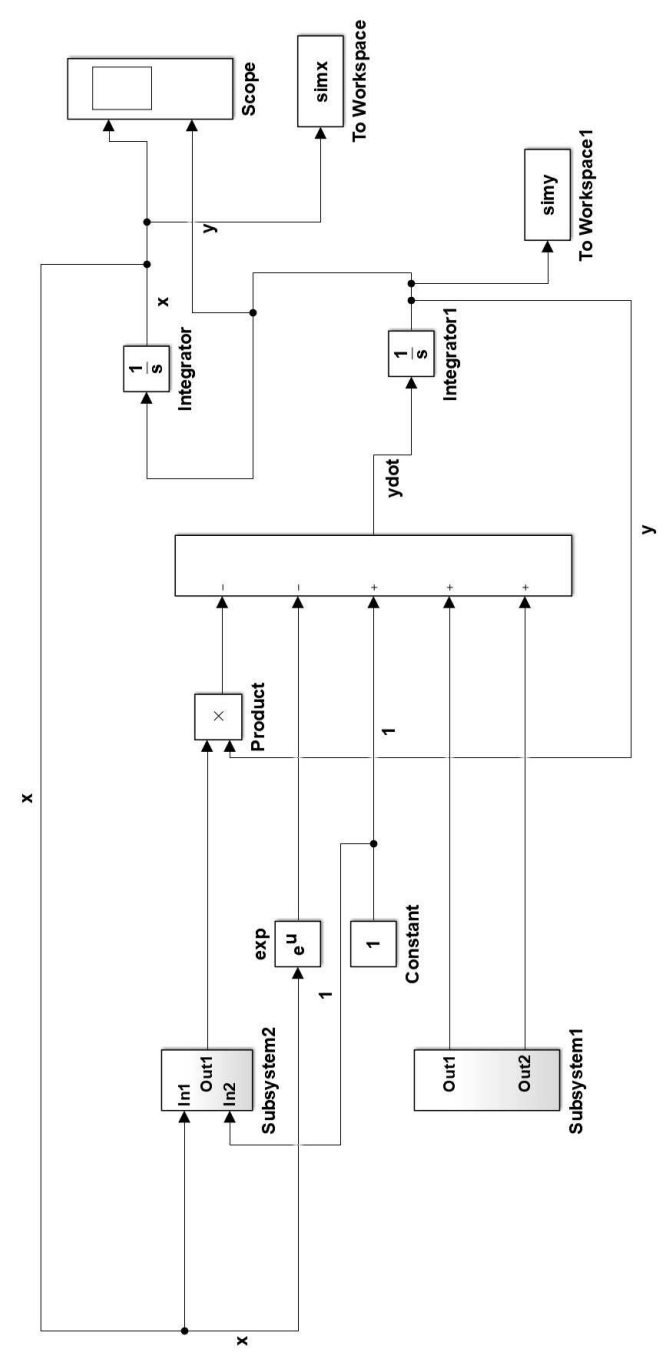

Fig. 10. Simulink implementation of the Toda oscillator system 1 strength of displacement-dependent periodic dissipation $\epsilon$ impacts significantly on the nature and occurrence of $\mathrm{VR}_{415}$ We emphasize that the present result is a departure from previous reports in which nonlinear dissipation enhances vibration-induced resonances as reported in [55|56|57]. The study showed that multiple VR is admissible in the model with the Toda potential for $\epsilon$ values in the region $\epsilon<-1.5$ and $\epsilon>0$ with the highest number of VR points observed for $\epsilon>\epsilon_{0}$, where $\epsilon_{0}$ is a threshold that depends on the high-frequency $\Omega$ values. Numerical approach was also em- ${ }^{420}$ ployed to confirm the theoretical analysis and very good agreement was achieved. This study provided insight into the conditions and the system parameter $\left(k_{0}\right.$ and $\left.\epsilon\right)$ regime for which one can achieve VR in the Toda oscillator model. The results have great potential for modelling modulated lasers 2122, class-B lasers 2324 and bad-cavity lasers 25. for laser output amplification. In this regard, we implemented the dual-frequency driven Toda oscillator mode ${ }^{425}$ using MATLAB-Simulink, compared the response output from the MATLAB-Simulink with theoretical results, and found excellent agreement. Finally, we remark that the ef-

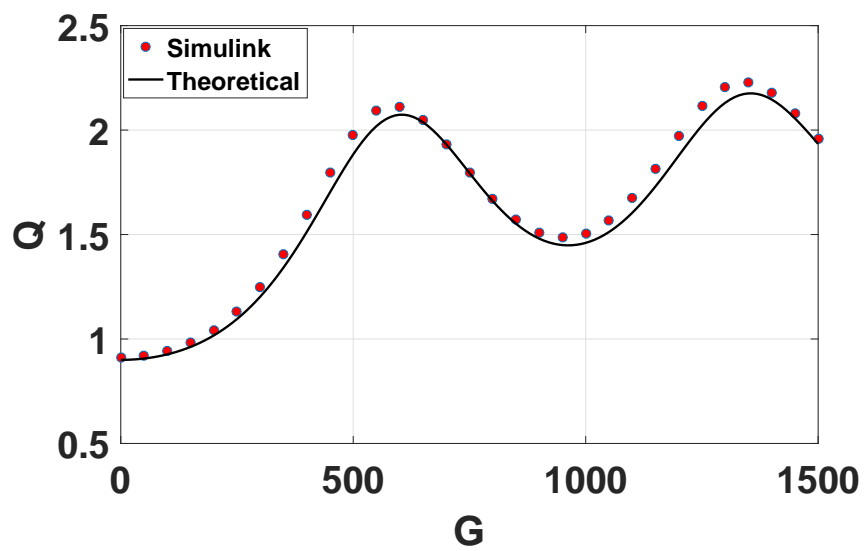

Fig. 11. The response amplitude $Q$ as a function of $G$ computed theoretically (continuous line) and Simulink simulation (red dots) of the Toda system Eq. (26) for $\epsilon=1.5, F=0.2, \omega^{2}=0.75, k_{0}=0.5$ and $\Omega=15$.

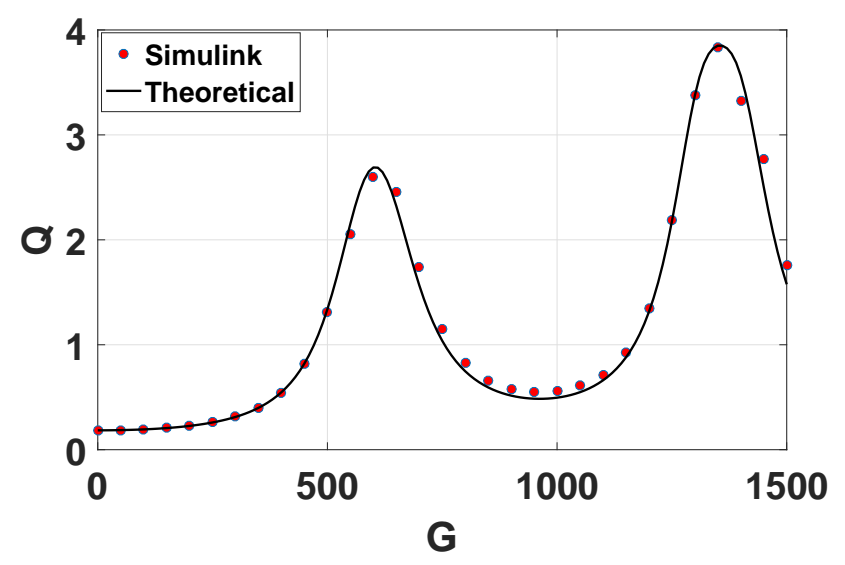

Fig. 12. The response amplitude $Q$ as a function of $G$ computed theoretically (continuous line) and Simulink simulation (red dots) of the Toda system Eq. (26) for $\epsilon=11.5, F=0.2, \omega^{2}=0.75, k_{0}=0.5$ and $\Omega=15$.

fects of dual-frequency driving presented here can be implemented in the context of the experimental configuration of class-B lasers presented by Cialdi et al. 24.

\section{Acknowledgments}

We acknowledge useful discussions with Dr. B. R. NanaNbendjo. The authors thank the anonymous Reviewers for their invaluable comments and suggestions that improved the quality and depth of this paper.

\section{References}

[1] M. Toda, Waves in nonlinear lattice, Progress of Theoretical Physics Supplement 45 (1970) 174-200.

[2] M. Toda, Studies of a non-linear lattice, Physics Reports 18 (1) (1975) $1-123$.

[3] M. Toda, Theory of Nonlinear Waves and Solitons, Kluwer, Dordrecht, 1989. 
[4] G. Teschl, Almost everything you always wanted to know about the Toda Equation, Jahresber. Deutsch. Math.-Verein. 103 (4)500 (2001) 149-162.

[5] W. Ebeling, U. Erdmann, J. Dunkel, M. Jenssen, Nonlinear dynamics and fluctuations of dissipative Toda chains, Journal of Statistical Physics 101 (1) (2000) 443-457.

[6] V. N. Likhachev, T. Y. Astakhova, G. A. Vinogradov505 Thermodynamics and ergodicity of finite one-dimensional Toda and Morse lattices, Physics Letters A 354 (4) (2006) $264-270$.

[7] M. A. Agrotis, P. A. Damianou, C. Sophocleous, The Toda lattice is super-integrable, Physica A 365 (1) (2006) 235 - 243.

[8] M. Hénon, Integrals of the Toda lattice, Phys. Rev. B $9(1974)_{510}$ 1921-1923.

[9] V. Muto, A. C. Scott, P. L. Christiansen, Thermally generated solitons in a Toda lattice model of DNA, Physics Letters A 136 (1-2) (1989) $33-36$.

[10] F. Mokross, H. Buttner, Thermally generated solitons in a Toda ${ }_{515}$ lattice model of DNA, J. Phys. C: Solid State Phys. 16 (23) (1983) 4539.

[11] S. Yomosa, Toda-lattice solitons in $\alpha$-helical proteins, J. Phys. Soc. Jpn. 53 (10) (1984) 3692-3698.

[12] M. Sun, S. Deng, D. Chen, The backlund transformation and 520 novel solutions for the Toda lattice, Chaos, Solitons \& Fractals 23 (4) (2005) $1169-1175$.

[13] X. Wen, N-fold Darboux Transformation and soliton solutions for Toda lattice equation, Reports on Mathematical Physics 68 (2) (2011) $211-223$.

[14] X. Xu, H. Yang, Y. Sun, Darboux transformation of the modified ${ }^{525}$ Toda lattice equation, Mod. Phys. Lett. B 20 (11) (2006) 641.

[15] C. Dai, Y. Wang, Exact travelling wave solutions of Toda lattice equations obtained via the exp-function method, Zeitschrift für Naturforschung A 63 (10-11) (2014) 657 - 662 .

[16] Z. Jia-Min, Hyperbolic function method for solving nonlinear 530 differential-different equations, Chinese Physics 14 (7) (2005) 1290 .

[17] T. Mizumachi, P. R.L., Asymptotic stability of Toda lattice solitons, Nonlinearity 21 (9) (2008) 2099.

[18] R. Mokhtari, Variational iteration method for solving nonlinear 535 differential- difference equations, International Journal of Nonlinear Sciences and Numerical Simulation 9 (1) (2011) 19 24.

[19] L. Wu, L. Xie, J. Zhang, Adomian decomposition method for nonlinear differential-difference equations, Communications in ${ }^{540}$ Nonlinear Science and Numerical Simulation 14 (1) (2009) 12 18.

[20] G. L. Oppo, A. Politi, Toda potential in laser equations, Zeitschrift für Physik B - Condensed Matter 59 (1985) 111-115.

[21] P. A. Braza, Phase jumps of $\pi$ in a laser with a periodically 545 modulated injected signal, Physica D 134 (4) (1999) $394-405$.

[22] Y. Khanin, Fundamentals of Laser Dynamics, Cambridge, 2006.

23] Y. Lien, S. de Vries, M. van Exter, J. Woerdman, Lasers as Toda oscillators, J. Opt. Soc. Am. B 19 (6) (2002) 1461-1466.

[24] S. Cialdi, F. Castelli, F. Prati, Lasers as Toda oscillators: An ${ }^{50}$ experimental confirmation, Optics Communications 287 (2013) $176-179$.

[25] T. Ogawa, Stochastic Toda-oscillator model of the bad-cavity laser, Phys. Rev. A 42 (1990) 4210-4225.

[26] T. Ogawa, Quasiperiodic instability and chaos in the bad-cavity555 laser with modulated inversion: Numerical analysis of a Toda oscillator system, Phys. Rev. A 37 (1988) 4286-4302.

[27] T. Kurz, W. Lauterborn, Bifurcation structure of the Toda oscillator, Physical Review A 37 (3) (1988) 1029-1031.

[28] B. K. Goswami, Observation of some new phenomena involving560 period tripling and period doubling, International Journal of Bifurcation and Chaos 05 (01) (1995) 303-312. T. Kapitaniak, Dynamics of three Toda oscillators with nonlinear unidirectional coupling, The European Physical Journal Speciab65 Topics 222 (10) (2013) 2429-2439.

[30] N. V. Stankevich, A. Dvorak, V. Astakhov, P. Jaros, M. Kapitaniak, P. Perlikowski, T. Kapitaniak, Chaos and hyperchaos in coupled antiphase driven Toda oscillators, Regular and Chaotic Dynamics 23 (1) (2018) 120-126.

[31] S.-Y. Kim, W. Lim, Universal mechanism for the intermittent route to strange nonchaotic attractors in quasiperiodically forced systems, J. Phys. A: Math \& Gen 37 (25) (2004) 6477-6489.

[32] P. S. Landa, P. V. E. McClintock, Vibrational resonance, J. Phys. A: Math. Gen. 33 (2000) 433-438.

[33] S. Rajasekar, M. A. F. Sanjuan, Nonlinear Resonances, Springer Series in Synergetics, Springer, Switzerland, 2016.

[34] H. C. Gerhardt, Significance of two frequency bands in long distance vocal communications in the green tree frog, Nature 261 (1976) 692-694.

[35] G. P. Agrawal, Fiber-Optic Communication Systems, Wiley, New York, 1992.

[36] D. C. Su, M. H. Chiu, C. D. Chen, Simple two-frequency laser, Precis. Eng. 18 (2-3) (1996) 161-163.

[37] A. O. Maksimov, On the subharmonic emission of gas bubbles under two-frequency excitation, Ultrasonics 35 (1997) 79-86.

[38] K. Harikrishnan, G. Ambika, Resonance phenomena in discrete systems with bichromatic input signal, EuroPhys. J. B 61 (3) (2008) 343-353.

[39] D. M. Ackermann, N. Bhadra, E. L. Foldes, K. L. Kilgore, Conduction block of whole nerve without onset firing using combined high frequency and direct current, Med. Biol. Eng. Comput. 49 (2) (2011) 241-251.

[40] C. Jeevarathinam, S. Rajasekar, M. A. F. Sanjuan, Vibrational resonance in groundwater-dependent plant ecosystems, Ecol. Complex. 15 (2013) 33-42.

[41] L. Ridolfi, P. D'Odorico, F. Laio, Vegetation dynamics induced by phreatophyte- water table interactions, J. Theor. Biol. 248 (2) (2007) 301-310.

[42] M. Gitterman, Bistable oscillator driven by two periodic fields, Journal of Physics A: Mathematical and General 34 (24) (2001) L355.

[43] I. I. Blekhman, P. S. Landa, Conjugate resonances and bifurcations in nonlinear systems under biharmonical excitation, Int. J. Nonlinear Mech. 39 (3) (2004) $421-426$.

[44] S. Jeyakumari, V. Chinnathanmbi, S. Rajasekar, M. A. F. Sanjuan, Analysis of vibrational resonance in a quintic oscillator, Chaos 19 (043128) (2009) 1-8.

[45] S. Jeyakumari, V. Chinnathanmbi, S. Rajasekar, M. A. F. Sanjuan, Single and multiple vibrational resonance in a quintic oscillator with monostable potentials, Physics Review E 80 (046608) (2009) 1-8.

[46] S. Jeyakumari, V. Chinnathanmbi, S. Rajasekar, M. A. F. Sanjuan, Novel vibrational resonance in multistable systems, Int. J. of Bifurcation and Chaos 21 (1) (2011) 275-286.

[47] B. Deng, J. Wang, X. Wei, K. M. Tsang, W. L. Chan, Vibrational resonance in neuron populations, Chaos 20 (1) (2010) 013113.

[48] C. Jeevarathinam, S. Rajasekar, M. A. F. Sanjuán, Effect of multiple time-delay on vibrational resonance, Chaos 23 (1) (2013) 013136.

[49] H. Liu, X. Liu, J. Yang, M. Sanjuan, G. Cheng, Detecting the weak high-frequency character signal by vibrational resonance in the Duffing oscillator, Nonlinear Dyn. 89 (4) (2017) 2621-2628.

[50] S. Rajasekar, J. Used, A. Wagemakers, M. A. F. Sanjuan, Vibrational resonance in biological nonlinear maps, Commun. Nonlinear Sci. Numer. Simulat. 17 (2012) 3435-3445.

[51] S. Ghosh, D. Ray, Nonlinear vibrational resonance, Physics Review E 88 (042904) (2013) 1-8.

[52] C. Wang, K. Yang, Vibrational resonance in bistable gene transcriptional regulatory system, Chinese J. of Physics 50 (4) (2012) 606-617.

[53] J. Shi, C. Huang, T. Dong, X. Zhang, High-frequency and lowfrequency effects on vibrational resonance in a synthetic gene network, Phys. Biol. 7 (3) (2010) 036006. 
[54] S. Morfu, M. Bordet, On the correlation between phase-locking modes and vibrational resonance in a neuronal model, Commun. Nonlinear Sci. Numer. Simulat. 55 (2018) 277-286.

[55] T. Djomo Mbong, M. Siewe Siewe, C. Tchawoua, The effect of nonlinear damping on vibrational resonance and chaotic behavior of a beam fixed at its two ends and prestressed, Commun. Nonlinear Sci. Numer. Simulat. 22 (2015) 228-243.

[56] T. O. Roy-Layinde, J. A. Laoye, O. Popoola, U. E. Vincent, Analysis of vibrational resonance in bi-harmonically driven plasma, Chaos 26 (9) (2016) 093117.

[57] T. O. Roy-Layinde, J. A. Laoye, O. O. Popoola, U. E. Vincent, P. V. E. McClintock, Vibrational resonance in an inhomogeneous medium with periodic dissipation, Phys. Rev. E 96 (2017) 032209 .

[58] U. E. Vincent, T. O. Roy-Layinde, O. O. Popoola, P. O. Adesina, P. V. E. McClintock, Vibrational resonance in an oscillator with an asymmetrical deformable potential, Phys. Rev. E 98 (2018) 062203.

[59] L. Ning, Z. Chen, Vibrational resonance analysis in a gene transcriptional regulatory system with two different forms of time-delays, Physica D 401 (2020) 132164.

[60] J. A. Laoye, T. O. Roy-Layinde, K. A. Omoteso, O. O. Popoola, U. E. Vincent, Vibrational resonance in a higher-order nonlinear damped oscillator with rough potential, Pramana 93 (6) (2019) 102.

[61] Z. Chen, L. Ning, Impact of depth and location of the wells on vibrational resonance in a triple-well system, Pramana J. Phys. 90 (4) (2018) 49.

[62] O. I. Olusola, O. P. Shomotun, U. E. Vincent, P. V. E. McClintock, Quantum vibrational resonance in a dual-frequency driven Tietz-Hua quantum well, Phys. Rev. E 101 (2020) 052216.

[63] P. Shibashis, R. Deb Shankar, Vibrational resonance in a driven two-level quantum system; linear and nonlinear response, Phil Trans Roy Soc. A 379 (2021) 20200231.

[64] T. O. Roy-Layinde, U. E. Vincent, S. A. Abolade, O. O. Popoola, J. A. Laoye, P. McClintock, Vibrational resonances in driven oscillators with position-dependent mass, Phil Trans Roy Soc. A 379 (2021) 20200227.

[65] Y. Ren, Y. Pan, F. Duan, Generalized energy detector for weak random signals via vibrational resonance, Physics Letters A 382 (12) (2018) $806-810$.

[66] P. Jia, C. Wu, J. Yang, M. Sanjuan, G. Liu, Improving the weak aperiodic signal by three kinds of vibrational resonance, Nonlinear Dyn. 91 (4) (2018) 2699-2713.

[67] V. Chizhevsky, Amplification of an autodyne signal in a bistable vertical-cavity surface-emitting laser with the use of a vibrational resonance, Technical Physics Letters 44 (1) (2018) 17-19.

[68] J.-H. Yang, M. A. F. Sanjuán, H.-G. Liu, Enhancing the weak signal with arbitrary high-frequency by vibrational resonance in fractional-order Duffing oscillators, ASME J. Comput. Nonlinear Dyn 12 (5) (2017) 051011.

[69] J.-R. Yang, C.-J. Wu, J.-H. Yang, H.-G. Liu, On the weak signal amplification by twice sampling vibrational resonance method in fractional Duffing oscillators, ASME J. Comput. Nonlinear Dyn 13 (2018) 031009.

[70] Y. Ren, Y. Pan, F. Duan, F. Chapeau-Blondeau, D. Abbott, Exploiting vibrational resonance in weak-signal detection, Phys. Rev. E 96 (2017) 022141.

[71] T. Klinker, W. Meyer-Ilse, W. Lauterborn, Period doubling and chaotic behavior in a driven Toda oscillator, Physics Letters A 101 (8) (1984) 371-375. 